NOTICE: This is the author's version of a work that was accepted for publication in Automatica. Changes resulting from the publishing process, such as peer review, editing, corrections, structural formatting, and other quality control mechanisms may not be reflected in this document. Changes may have been made to this work since it was submitted for publication. A definitive version was subsequently published in Automatica, Vol. 44, Issue 11 (2008). doi: 10.1016/j.automatica.2008.04.011 


\title{
Optimal control problems with multiple characteristic time points in the objective and constraints *
}

\author{
R. C. Loxton ${ }^{\mathrm{a}}$, K. L. Teo ${ }^{\mathrm{a}}$, V. Rehbock ${ }^{\mathrm{a}}$ \\ ${ }^{a}$ Department of Mathematics and Statistics, Curtin University of Technology, Perth, Australia
}

\begin{abstract}
In this paper, we develop a computational method for a class of optimal control problems where the objective and constraint functionals depend on two or more discrete time points. These time points can be either fixed or variable. Using the control parametrization technique and a time scaling transformation, this type of optimal control problem is approximated by a sequence of approximate optimal parameter selection problems. Each of these approximate problems can be viewed as a finite dimensional optimization problem. New gradient formulae for the cost and constraint functions are derived. With these gradient formulae, standard gradient-based optimization methods can be applied to solve each approximate optimal parameter selection problem. For illustration, two numerical examples are solved.
\end{abstract}

Key words: Control parametrization; Optimal control computation; Non-linear optimal control; Constrained optimal control; Non-linear programming.

\section{Introduction}

In this paper, we consider a general class of optimal control problems where the objective and constraint functionals depend on two or more discrete time points. These time points are known as characteristic times and such problems are referred to as multiple characteristic time optimal control problems. An example arises in the study of drug administration for cancer chemotherapy. Martin \& Teo (1994) and Martin (1992) examined the delivery schedule for this type of anti-cancer drug from an optimal control viewpoint. The planning horizon is partitioned into a set of intervals and the size of the cancer tumour is required to decrease sufficiently over each interval. This stipulation forces the tumour size to decrease at, or faster than, a given rate and leads to a set of inequality constraints. Each of these inequality constraints depends on two characteristic times.

Consider another important application. Suppose we wish to construct a dynamic model to simulate a physi-

\footnotetext{
^ This paper was not presented at any IFAC meeting. Research supported by the National Natural Science Foundation of China under Grant 60704003 and a grant from the Australian Research Council.

Email addresses: R.Loxton@curtin.edu.au (R. C.

Loxton), K.L.Teo@curtin.edu. au (K. L. Teo), rehbock@maths.curtin.edu.au (V. Rehbock).
}

cal system, where some of the model parameters are to be identified. The problem of choosing values for these unknown parameters in such a way that the solution of the dynamic model will best fit experimental data at a set of sample points can be formulated as an optimal parameter selection problem with the objective function depending on many characteristic times. This technique has recently been applied to the study of crystallization processes; see Livk, Pohar \& Ilievski (1999) and Li, Livk \& Ilievski (2001), for example. However, finite difference approximations are often employed to generate the gradients required by the underlying optimization procedure. Thus, convergence can be poor or non-existent.

Martin \& Teo (1994) and Martin (1992) solved the fixed multiple characteristic time optimal control problem numerically using the classical control parametrization scheme (see Teo, Goh \& Wong (1991)). Relevant gradient formulae were derived. On this basis, a gradient-based optimization technique, such as sequential quadratic programming (SQP) (see Nocedal \& Wright (1999)), is used to generate a numerical solution. However, computation of the required gradients involves the integration of a system of state differential equations forward in time, followed by the integration of corresponding systems of costate differential equations with jumps backwards in time. The presence of the jump conditions makes the application of this technique cumbersome. Moreover, the state and costate 
systems are solved in opposite directions using an adaptive integration scheme. Thus, it is impossible to ensure that the state and costate knot sets coincide. Since each costate system depends on the solution of the state system, an appropriate interpolation method needs to be invoked. Hence, the accuracy of the gradients obtained is compromised.

Teo, Lee, Jennings, Wang \& Liu (2002) considered the case where the characteristic times are variable. Control parametrization was again used - this time, in conjunction with two time scaling transformations - and an approximate problem with fixed characteristic times was derived. This approximate problem can be solved using the gradient formulae previously developed by Martin \& Teo (1994).

In this paper, we derive alternative gradient formulae using an approach first introduced by Vincent \& Grantham (1981) and extended by Kaya \& Noakes (2003). Our gradient computation scheme is applicable to both fixed and variable multiple characteristic time optimal control problems and relies on the continuous integration of differential equations forward in time. Thus, it has two important advantages over the existing scheme: the complexities involved in dealing with a discontinuous costate system are avoided; and, more importantly, no interpolation of the state is necessary if a variable step size integration method is used to solve the relevant differential equations.

\section{Problem Statement}

We will introduce two optimal control problems: one with fixed characteristic times, and the other with characteristic times as optimization parameters. Consider a process evolving over the fixed time horizon $[0, T]$ according to the dynamical system

$$
\dot{\mathbf{x}}(t)=\mathbf{f}(\mathbf{x}(t), \mathbf{u}(t))
$$

with the initial condition

$$
\mathbf{x}(0)=\mathbf{x}^{\mathbf{0}}
$$

Here, $\mathbf{x}(t) \in \mathbb{R}^{n}$ is the state of the process at time $t$, $\mathbf{u}(t) \in \mathbb{R}^{r}$ is the control input applied at time $t$, and $\mathbf{f}: \mathbb{R}^{n} \times \mathbb{R}^{r} \rightarrow \mathbb{R}^{n}$ is a given function continuously differentiable with respect to each of its arguments. Define

$$
U=\left\{\mathbf{v}=\left[v_{i}\right] \in \mathbb{R}^{r}: v_{i}^{L} \leq v_{i} \leq v_{i}^{U}, i=1, \ldots, r\right\},
$$

where $v_{i}^{L}$ and $v_{i}^{U}, i=1, \ldots, r$, are given constants such that $v_{i}^{L}<v_{i}^{U}$. Any Borel measurable function $\mathbf{u}:[0, T] \rightarrow \mathbb{R}^{r}$ such that $\mathbf{u}(t) \in U$ almost everywhere in $[0, T]$ is said to be an admissible control. Let $\mathcal{U}$ be the class of all such admissible controls.
For a given $\mathbf{u} \in \mathcal{U}$, let $\mathbf{x}(\cdot \mid \mathbf{u})$ be the corresponding solution to (1) that satisfies the initial condition (2). We suppose that, for each $i=1, \ldots, N$, the following inequality constraint is required to be satisfied:

$$
\begin{aligned}
g_{i}(\mathbf{u})=\Phi_{i}\left(\mathbf{x}\left(\tau_{1} \mid \mathbf{u}\right)\right. & \left., \ldots, \mathbf{x}\left(\tau_{m} \mid \mathbf{u}\right)\right) \\
& +\int_{0}^{T} \mathcal{L}_{i}(\mathbf{x}(t \mid \mathbf{u}), \mathbf{u}(t)) d t \geq 0,
\end{aligned}
$$

where $\boldsymbol{\tau}=\left[\tau_{1}, \ldots, \tau_{m}\right]^{T} \in \mathbb{R}^{m}$ is a fixed vector such that $0<\tau_{1}<\cdots<\tau_{m}<T$. Each $\tau_{i}, i=1, \ldots, m$, is called a characteristic time. Moreover, we assume that, for each $i=0, \ldots, N, \mathcal{L}_{i}: \mathbb{R}^{n} \times \mathbb{R}^{r} \rightarrow \mathbb{R}$ and $\Phi_{i}: \mathbb{R}^{n m} \rightarrow \mathbb{R}$ are given functions continuously differentiable with respect to each of their arguments.

Denote by $\mathcal{F}$ the set of all $\mathbf{u} \in \mathcal{U}$ satisfying (3) for each $i=1, \ldots, N$. With this groundwork, we are in a position to introduce our first optimal control problem.

Problem $\left(\mathbf{P}_{\mathbf{1}}\right)$. Given the system (1) and the initial condition (2), find a $\mathbf{u} \in \mathcal{F}$ such that the cost functional

$$
\begin{aligned}
g_{0}(\mathbf{u})=\Phi_{0}\left(\mathbf{x}\left(\tau_{1} \mid \mathbf{u}\right),\right. & \left.\ldots, \mathbf{x}\left(\tau_{m} \mid \mathbf{u}\right)\right) \\
& +\int_{0}^{T} \mathcal{L}_{0}(\mathbf{x}(t \mid \mathbf{u}), \mathbf{u}(t)) d t
\end{aligned}
$$

is minimized over $\mathcal{F}$.

In Problem $\left(P_{1}\right)$ above, the characteristic times appearing in the objective and constraint functionals are fixed. It is not difficult, however, to envisage a practical scenario where these are variable. Suppose now then, that $\tau$ is not fixed and is actually a parameter vector to be chosen optimally from the set

$$
\mathcal{S}=\left\{\boldsymbol{\tau}=\left[\tau_{i}\right] \in \mathbb{R}^{m}: \tau_{i}^{L} \leq \tau_{i} \leq \tau_{i}^{U}, i=1, \ldots, m\right\},
$$

where $\tau_{i}^{L}$ and $\tau_{i}^{U}, i=1, \ldots, m$, are given constants such that $0 \leq \tau_{1}^{L} \leq \tau_{1}^{U} \leq \cdots \leq \tau_{m}^{L} \leq \tau_{m}^{U} \leq T$. Here, the left hand side of (3) should be replaced by $g_{i}(\mathbf{u}, \boldsymbol{\tau})$. Let $\mathcal{G}$ be the set of combined elements $(\mathbf{u}, \boldsymbol{\tau}) \in \mathcal{U} \times \mathcal{S}$ that satisfy all of these constraints. Our second optimal control problem is stated as follows.

Problem $\left(\mathbf{P}_{\mathbf{2}}\right)$. Given the system (1) and the initial condition (2), find a combined element $(\mathbf{u}, \boldsymbol{\tau}) \in \mathcal{G}$ such that the cost functional (4), with the left hand side replaced by $g_{0}(\mathbf{u}, \boldsymbol{\tau})$, is minimized over $\mathcal{G}$.

\section{Control Parametrization}

To solve Problem $\left(P_{1}\right)$ or $\left(P_{2}\right)$ numerically, we apply the classical control parametrization scheme. That is, the control is approximated by a piecewise constant function with a finite number of discontinuities. 
Let $\tau_{0}=0$ and $\tau_{m+1}=T$. Within each subinterval $\left[\tau_{k-1}, \tau_{k}\right], k=1, \ldots, m+1$, allow the approximate control to switch at $t_{j}^{k}, j=1, \ldots, p_{k}-1$ (these switching times are labeled in non-decreasing order). In addition, allow the approximate control to switch at each characteristic time. For each $k=1, \ldots, m+1$, denote $t_{0}^{k}=\tau_{k-1}$ and $t_{p_{k}}^{k}=\tau_{k}$. Then $\left[t_{j-1}^{k}, t_{j}^{k}\right), k=1, \ldots, m+1, j=$ $1, \ldots, p_{k}$, is a partition of the time horizon. Label each subinterval with the index $\beta_{j}^{k}=\sum_{l=1}^{k-1} p_{l}+j$. With this numeration scheme, the subintervals are labeled from left to right along the time axis in chronological order. Thus, the approximate control can be expressed as

$$
\mathbf{u}^{p}(t)=\sum_{k=1}^{m+1} \sum_{j=1}^{p_{k}} \boldsymbol{\sigma}^{\beta_{j}^{k}} \chi_{\left[t_{j-1}^{k}, t_{j}^{k}\right)}(t),
$$

where

$$
\chi_{I}(t)=\left\{\begin{array}{l}
1, \text { if } t \in I, \\
0, \text { otherwise, }
\end{array}\right.
$$

and

$$
p=\sum_{k=1}^{m+1} p_{k}
$$

For $\mathbf{u}^{p}$ to be an admissible control, we must have $\boldsymbol{\sigma}^{l}=$ $\left[\sigma_{1}^{l}, \ldots, \sigma_{r}^{l}\right]^{T} \in U$ for $l=1, \ldots, p$. Denote by $\Xi$ the set of all $\boldsymbol{\sigma}=\left[\left(\boldsymbol{\sigma}^{1}\right)^{T}, \ldots,\left(\boldsymbol{\sigma}^{p}\right)^{T}\right]^{T} \in \mathbb{R}^{p r}$ that satisfy this requirement.

To obtain an approximate optimal control, the parameters in (5) must be chosen to minimize the relevant performance index given in Section 2. However, gradientbased optimization algorithms are not effective for determining the optimal switching times (for details, see Lee, Teo, Rehbock \& Jennings (1997)). Thus, we employ the time scaling transformation introduced in Lee et al. (1997) to map these switching times into a set of fixed time points on a new time horizon. Firstly, let

$$
\Theta=\left\{\boldsymbol{\theta}=\left[\theta_{1}, \ldots, \theta_{p}\right]^{T} \in \mathbb{R}^{p}: \theta_{l} \geq 0, l=1, \ldots, p\right\} .
$$

Then, for any $\boldsymbol{\theta} \in \Theta$ such that

$$
\sum_{l=1}^{p} \frac{\theta_{l}}{p}=T
$$

we can define a monotonic transformation from $t \in[0, T]$ to a new time scale $s \in[0,1]$ by

$$
\frac{d t(s)}{d s}=v^{p}(s)=\sum_{k=1}^{p} \theta_{k} \chi_{\left[\frac{k-1}{p}, \frac{k}{p}\right)}(s)
$$

with the initial condition

$$
t(0)=0
$$

By integrating (6) and using (7), it is easy to see that, for $s \in\left[\frac{q-1}{p}, \frac{q}{p}\right)$,

$$
t(s)=\sum_{l=1}^{q-1} \frac{\theta_{l}}{p}+\frac{\theta_{q}}{p}(p s-q+1) .
$$

In the new time horizon, the control (5) has fixed switching times at $s=\frac{1}{p}, \ldots, \frac{p-1}{p}$ and can be expressed as:

$$
\tilde{\mathbf{u}}^{p}(s)=\mathbf{u}^{p}(t(s))=\sum_{k=1}^{p} \boldsymbol{\sigma}^{k} \chi_{\left[\frac{k-1}{p}, \frac{k}{p}\right)}(s) .
$$

For each $i=1, \ldots, m+1$, define

$$
\kappa_{i}=\sum_{l=1}^{i} p_{l} \quad \text { and } \quad \zeta_{i}=\frac{\kappa_{i}}{p}
$$

Then the characteristic times can be recovered from (8) by

$$
\tau_{i}=t\left(\zeta_{i}\right)=\sum_{l=1}^{\kappa_{i}} \frac{\theta_{l}}{p}, \quad i=1, \ldots, m+1 .
$$

Finally, applying the transformation to the dynamics (1) yields

$$
\dot{\mathbf{y}}(s)=v^{p}(s) \tilde{\mathbf{f}}(\mathbf{y}(s), \boldsymbol{\sigma}),
$$

with the initial condition

$$
\mathbf{y}(0)=\mathbf{x}^{\mathbf{0}}
$$

where $\mathbf{y}(s)=\mathbf{x}(t(s))$ and $\tilde{\mathbf{f}}(\mathbf{y}(s), \boldsymbol{\sigma})=\mathbf{f}\left(\mathbf{x}(t(s)), \tilde{\mathbf{u}}^{p}(s)\right)$.

Given a $(\boldsymbol{\sigma}, \boldsymbol{\theta}) \in \Xi \times \Theta$, one can solve the system of differential equations (10) using the initial condition (11) to obtain $\mathbf{y}(s \mid \boldsymbol{\sigma}, \boldsymbol{\theta})$. Then, the objective and constraint functionals $g_{i}, i=0, \ldots, N$, for Problems $\left(P_{1}\right)$ and $\left(P_{2}\right)$ collectively become

$$
\begin{aligned}
G_{i}(\boldsymbol{\sigma}, \boldsymbol{\theta})= & \Phi_{i}\left(\mathbf{y}\left(\zeta_{1} \mid \boldsymbol{\sigma}, \boldsymbol{\theta}\right), \ldots, \mathbf{y}\left(\zeta_{m} \mid \boldsymbol{\sigma}, \boldsymbol{\theta}\right)\right) \\
& +\int_{0}^{1} v^{p}(s) \tilde{\mathcal{L}}_{i}(\mathbf{y}(s \mid \boldsymbol{\sigma}, \boldsymbol{\theta}), \boldsymbol{\sigma}) d s
\end{aligned}
$$

where $\tilde{\mathcal{L}}_{i}$ is obtained from $\mathcal{L}_{i}$ in the same manner as $\tilde{\mathbf{f}}$ is obtained from $\mathbf{f}$.

Finally, defining

$$
\Upsilon=\left\{(\boldsymbol{\sigma}, \boldsymbol{\theta}) \in \Xi \times \Theta: G_{i}(\boldsymbol{\sigma}, \boldsymbol{\theta}) \geq 0, i=1, \ldots, N\right\},
$$

we can introduce approximate problems corresponding to Problems $\left(P_{1}\right)$ and $\left(P_{2}\right)$ as follows.

Problem $\left(\mathbf{P}_{\mathbf{1}}(\mathbf{p})\right)$. Given the system (10) and the initial condition (11), find a combined element $(\boldsymbol{\sigma}, \boldsymbol{\theta}) \in \Upsilon$ 
such that the cost function $G_{0}(\boldsymbol{\sigma}, \boldsymbol{\theta})$ is minimized over $\Upsilon$ subject to

$$
\sum_{l=1}^{\kappa_{i}} \frac{\theta_{l}}{p}=\tau_{i}, \quad i=1, \ldots, m+1
$$

Problem $\left(\mathbf{P}_{\mathbf{2}}(\mathbf{p})\right)$. Given the system (10) and the initial condition (11), find a combined element $(\boldsymbol{\sigma}, \boldsymbol{\theta}) \in \Upsilon$ such that the cost function $G_{0}(\boldsymbol{\sigma}, \boldsymbol{\theta})$ is minimized over $\Upsilon$ subject to

$$
\sum_{l=1}^{p} \frac{\theta_{l}}{p}=T
$$

and

$$
\tau_{i}^{L} \leq \sum_{l=1}^{\kappa_{i}} \frac{\theta_{l}}{p} \leq \tau_{i}^{U}, \quad i=1, \ldots, m
$$

$\boldsymbol{R e m a r k} 1$. For any $(\boldsymbol{\sigma}, \boldsymbol{\theta}) \in \Upsilon$, the corresponding piecewise constant control $\mathbf{u}^{p}$ can be readily constructed according to (5) with

$$
t_{j}^{k}=\sum_{l=1}^{\kappa_{k-1}+j} \frac{\theta_{l}}{p}, \quad k=1, \ldots, m+1, j=0, \ldots, p_{k} .
$$

Similarly, the corresponding characteristic times can be constructed from (9).

Problems $\left(P_{1}(p)\right)$ and $\left(P_{2}(p)\right)$ can be viewed as nonlinear optimization problems where the cost function is to be minimized subject to a set of constraints. A gradient-based optimization procedure such as SQP can be used to solve these approximate problems if formulae for calculating the required gradients are available. These formulae will be derived in the next section. Firstly, however, we present the following convergence results. The proofs are similar to the arguments used in Chapter 4 of Martin \& Teo (1994) and Chapter 6 of Teo et al. (1991).

Theorem 1. Suppose that $\mathbf{u}^{*}$ is an optimal control for Problem $\left(P_{1}\right)$. Let $\left(\boldsymbol{\sigma}^{p, *}, \boldsymbol{\theta}^{p, *}\right)$ be a combined optimal element for Problem $\left(P_{1}(p)\right)$ and let $\mathbf{u}^{p \text {,* }}$ be the corresponding piecewise constant control constructed from $\boldsymbol{\sigma}^{p, *}$ and $\boldsymbol{\theta}^{p, *}$ as described in Remark 1. Define $\alpha=$ $\min \left\{p_{1}, \ldots, p_{m+1}\right\}$. Then

$$
\lim _{\alpha \rightarrow \infty} g_{0}\left(\mathbf{u}^{p, *}\right)=g_{0}\left(\mathbf{u}^{*}\right) .
$$

Theorem 2. Let $\mathbf{u}^{*}, \mathbf{u}^{p, *}$ and $\alpha$ be as defined in Theorem 1. Suppose that the sequence $\left\{\mathbf{u}^{p, *}\right\}_{\alpha=1}^{\infty}$ converges to a control $\overline{\mathbf{u}}$ almost everywhere in $[0, T]$. Then $\overline{\mathbf{u}}$ is an optimal control for Problem $\left(P_{1}\right)$.
Remark 2. Results analogous to Theorems 1 and 2 are also valid for Problem $\left(P_{2}\right)$ and approximate Problems $\left(P_{2}(p)\right)$.

\section{Gradient Formulae}

Let $(\boldsymbol{\sigma}, \boldsymbol{\theta}) \in \Xi \times \Theta$. For each $k=1, \ldots, p$ and $j=$ $1, \ldots, r$, consider the following system of $n$ auxiliary differential equations on $s \in\left[\frac{q-1}{p}, \frac{q}{p}\right), q=1, \ldots, p$ :

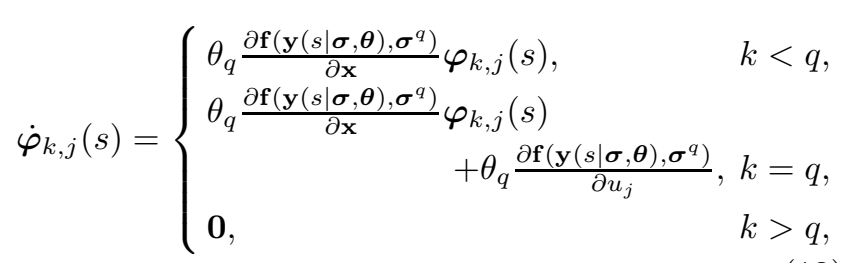

with

$$
\boldsymbol{\varphi}_{k, j}(0)=\mathbf{0}
$$

Similarly, for each $k=1, \ldots, p$, consider the following system on $s \in\left[\frac{q-1}{p}, \frac{q}{p}\right), q=1, \ldots, p$ :

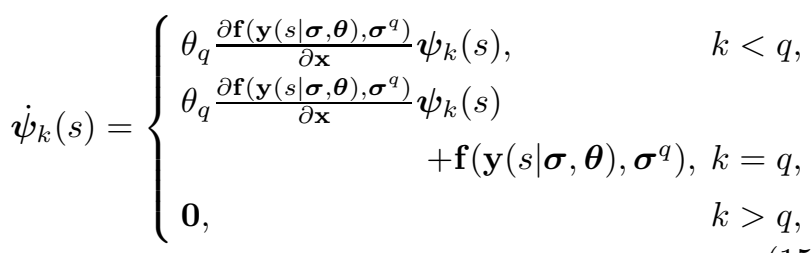

with

$$
\boldsymbol{\psi}_{k}(0)=\mathbf{0} .
$$

The gradients of the cost and constraint functions for the approximate problems are furnished in the following theorem.

Theorem 3. For each $i=0, \ldots, N$, we have

$$
\begin{aligned}
\frac{\partial G_{i}(\boldsymbol{\sigma}, \boldsymbol{\theta})}{\partial \sigma_{j}^{k}}= & \sum_{l=1}^{m} \frac{\partial \Phi_{i}\left(\mathbf{y}\left(\zeta_{1} \mid \boldsymbol{\sigma}, \boldsymbol{\theta}\right), \ldots, \mathbf{y}\left(\zeta_{m} \mid \boldsymbol{\sigma}, \boldsymbol{\theta}\right)\right)}{\partial \mathbf{x}\left(\tau_{l}\right)} \boldsymbol{\varphi}_{k, j}\left(\zeta_{l}\right) \\
& +\sum_{l=1}^{p} \int_{(l-1) / p}^{l / p} \theta_{l} \frac{\partial \mathcal{L}_{i}\left(\mathbf{y}(s \mid \boldsymbol{\sigma}, \boldsymbol{\theta}), \boldsymbol{\sigma}^{l}\right)}{\partial \mathbf{x}} \boldsymbol{\varphi}_{k, j}(s) d s \\
& +\int_{(k-1) / p}^{k / p} \theta_{k} \frac{\partial \mathcal{L}_{i}\left(\mathbf{y}(s \mid \boldsymbol{\sigma}, \boldsymbol{\theta}), \boldsymbol{\sigma}^{k}\right)}{\partial u_{j}} d s
\end{aligned}
$$

for $k=1, \ldots, p, j=1, \ldots, r$; and

$$
\begin{aligned}
\frac{\partial G_{i}(\boldsymbol{\sigma}, \boldsymbol{\theta})}{\partial \theta_{k}}= & \sum_{l=1}^{m} \frac{\partial \Phi_{i}\left(\mathbf{y}\left(\zeta_{1} \mid \boldsymbol{\sigma}, \boldsymbol{\theta}\right), \ldots, \mathbf{y}\left(\zeta_{m} \mid \boldsymbol{\sigma}, \boldsymbol{\theta}\right)\right)}{\partial \mathbf{x}\left(\tau_{l}\right)} \boldsymbol{\psi}_{k}\left(\zeta_{l}\right) \\
& +\sum_{l=1}^{p} \int_{(l-1) / p}^{l / p} \theta_{l} \frac{\partial \mathcal{L}_{i}\left(\mathbf{y}(s \mid \boldsymbol{\sigma}, \boldsymbol{\theta}), \boldsymbol{\sigma}^{l}\right)}{\partial \mathbf{x}} \boldsymbol{\psi}_{k}(s) d s \\
& +\int_{(k-1) / p}^{k / p} \mathcal{L}_{i}\left(\mathbf{y}(s \mid \boldsymbol{\sigma}, \boldsymbol{\theta}), \boldsymbol{\sigma}^{k}\right) d s
\end{aligned}
$$

for $k=1, \ldots, p$. 
Proof. Note that equation (12) can be written as

$$
\begin{aligned}
G_{i}(\boldsymbol{\sigma}, \boldsymbol{\theta})= & \Phi_{i}\left(\mathbf{y}\left(\zeta_{1} \mid \boldsymbol{\sigma}, \boldsymbol{\theta}\right), \ldots, \mathbf{y}\left(\zeta_{m} \mid \boldsymbol{\sigma}, \boldsymbol{\theta}\right)\right) \\
& +\sum_{l=1}^{p} \int_{(l-1) / p}^{l / p} \theta_{l} \mathcal{L}_{i}\left(\mathbf{y}(s \mid \boldsymbol{\sigma}, \boldsymbol{\theta}), \boldsymbol{\sigma}^{l}\right) d s
\end{aligned}
$$

For any $k=1, \ldots, p$ and $j=1, \ldots, r$, applying the chain rule in conjunction with the Leibniz rule to (17) yields

$$
\begin{aligned}
\frac{\partial G_{i}(\boldsymbol{\sigma}, \boldsymbol{\theta})}{\partial \sigma_{j}^{k}}= & \sum_{l=1}^{m} \frac{\partial \Phi_{i}\left(\mathbf{y}\left(\zeta_{1} \mid \boldsymbol{\sigma}, \boldsymbol{\theta}\right), \ldots, \mathbf{y}\left(\zeta_{m} \mid \boldsymbol{\sigma}, \boldsymbol{\theta}\right)\right)}{\partial \mathbf{x}\left(\tau_{l}\right)} \frac{\partial \mathbf{y}\left(\zeta_{l} \mid \boldsymbol{\sigma}, \boldsymbol{\theta}\right)}{\partial \sigma_{j}^{k}} \\
& +\sum_{l=1}^{p} \int_{(l-1) / p}^{l / p} \theta_{l} \frac{\partial \mathcal{L}_{i}\left(\mathbf{y}(s \mid \boldsymbol{\sigma}, \boldsymbol{\theta}), \boldsymbol{\sigma}^{l}\right)}{\partial \mathbf{x}} \frac{\partial \mathbf{y}(s \mid \boldsymbol{\sigma}, \boldsymbol{\theta})}{\partial \sigma_{j}^{k}} d s \\
& +\int_{(k-1) / p}^{k / p} \theta_{k} \frac{\partial \mathcal{L}_{i}\left(\mathbf{y}(s \mid \boldsymbol{\sigma}, \boldsymbol{\theta}), \boldsymbol{\sigma}^{k}\right)}{\partial u_{j}} d s .
\end{aligned}
$$

Now, given a $(\boldsymbol{\sigma}, \boldsymbol{\theta}) \in \Xi \times \Theta$, it follows from (10) that, for any $s \in\left[\frac{q-1}{p}, \frac{q}{p}\right]$,

$$
\begin{aligned}
\mathbf{y}(s \mid \boldsymbol{\sigma}, \boldsymbol{\theta})= & \mathbf{y}((q-1) / p \mid \boldsymbol{\sigma}, \boldsymbol{\theta}) \\
& +\int_{(q-1) / p}^{s} \theta_{q} \mathbf{f}\left(\mathbf{y}(\eta \mid \boldsymbol{\sigma}, \boldsymbol{\theta}), \boldsymbol{\sigma}^{q}\right) d \eta .
\end{aligned}
$$

Using the Leibniz rule to differentiate (19) with respect to $\sigma_{j}^{k}, k=1, \ldots, q-1$ and $j=1, \ldots, r$, gives

$$
\begin{aligned}
\frac{\partial \mathbf{y}(s \mid \boldsymbol{\sigma}, \boldsymbol{\theta})}{\partial \sigma_{j}^{k}}= & \frac{\partial \mathbf{y}((q-1) / p \mid \boldsymbol{\sigma}, \boldsymbol{\theta})}{\partial \sigma_{j}^{k}} \\
& +\int_{(q-1) / p}^{s} \theta_{q} \frac{\partial \mathbf{f}\left(\mathbf{y}(\eta \mid \boldsymbol{\sigma}, \boldsymbol{\theta}), \boldsymbol{\sigma}^{q}\right)}{\partial \mathbf{x}} \frac{\partial \mathbf{y}(\eta \mid \boldsymbol{\sigma}, \boldsymbol{\theta})}{\partial \sigma_{j}^{k}} d \eta
\end{aligned}
$$

For $k=q$, following the same procedure furnishes

$$
\begin{aligned}
\frac{\partial \mathbf{y}(s \mid \boldsymbol{\sigma}, \boldsymbol{\theta})}{\partial \sigma_{j}^{k}}= & \frac{\partial \mathbf{y}((q-1) / p \mid \boldsymbol{\sigma}, \boldsymbol{\theta})}{\partial \sigma_{j}^{k}} \\
& +\int_{(q-1) / p}^{s} \theta_{q} \frac{\partial \mathbf{f}\left(\mathbf{y}(\eta \mid \boldsymbol{\sigma}, \boldsymbol{\theta}), \boldsymbol{\sigma}^{q}\right)}{\partial \mathbf{x}} \frac{\partial \mathbf{y}(\eta \mid \boldsymbol{\sigma}, \boldsymbol{\theta})}{\partial \sigma_{j}^{k}} d \eta \\
& +\int_{(q-1) / p}^{s} \theta_{q} \frac{\partial \mathbf{f}\left(\mathbf{y}(\eta \mid \boldsymbol{\sigma}, \boldsymbol{\theta}), \boldsymbol{\sigma}^{q}\right)}{\partial u_{j}} d \eta
\end{aligned}
$$

Clearly, we have

$$
\frac{\partial \mathbf{y}(s \mid \boldsymbol{\sigma}, \boldsymbol{\theta})}{\partial \sigma_{j}^{k}}=\mathbf{0}
$$

for all $k>q, j=1, \ldots, r$. Now, by differentiating (20), (21) and (22), it is evident that $\frac{\partial \mathbf{y}(s \mid \boldsymbol{\sigma}, \boldsymbol{\theta})}{\partial \sigma_{j}^{k}}$ satisfies the system of differential equations (13) with the initial conditions (14). From the theory of differential equations, the solution to the system (13) endowed with the initial condition (14) is unique. On this basis, equation (18) is identical to the first formula in the statement of the theorem. The derivation of the other formula is similar.

On the basis of Theorem 3, we present an algorithm for computing the objective and constraint functions as well as their respective gradients as follows.
Algorithm 1. Input $\left(\boldsymbol{\sigma}^{0}, \boldsymbol{\theta}^{0}\right) \in \Xi \times \Theta$.

(1) Solve the system of differential equations consisting of (10), (13) and (15) with the initial conditions (11), (14) and (16) using any numerical integration scheme.

(2) Use $\mathbf{y}\left(\cdot \mid \boldsymbol{\sigma}^{0}, \boldsymbol{\theta}^{0}\right)$ with equation (17) to calculate $G_{i}\left(\boldsymbol{\sigma}^{0}, \boldsymbol{\theta}^{0}\right), i=0, \ldots, N$.

(3) Use $\mathbf{y}\left(\cdot \mid \boldsymbol{\sigma}^{0}, \boldsymbol{\theta}^{0}\right), \boldsymbol{\varphi}_{j, k}\left(\cdot \mid \boldsymbol{\sigma}^{0}, \boldsymbol{\theta}^{0}\right)$ and $\boldsymbol{\psi}_{k}\left(\cdot \mid \boldsymbol{\sigma}^{0}, \boldsymbol{\theta}^{0}\right)$ with the formulae in Theorem 3 to compute the gradients of $G_{i}, i=0, \ldots, N$, at $\left(\boldsymbol{\sigma}^{0}, \boldsymbol{\theta}^{0}\right)$.

Remark 3. Note that costate-based methods for gradient computation require the solution of $N+1$ costate systems of differential equations. In Algorithm 1, $p+p r$ systems of auxiliary differential equations are solved. Thus, Algorithm 1 is more efficient for problems with constraints outnumbering the optimization parameters.

Remark 4. Note that the above analysis can be extended to more general problems with system parameters appearing in the dynamical system and/or constraint and objective functionals. This is a simple extension and is not included here for the sake of brevity.

We are now in a position to solve Problem $\left(P_{1}\right)$ or $\left(P_{2}\right)$ numerically. To begin, we approximate Problem $\left(P_{1}\right)$ or $\left(P_{2}\right)$ by the corresponding optimal parameter selection Problem $\left(P_{1}(p)\right)$ or $\left(P_{2}(p)\right)$. By employing Algorithm 1 , the approximate problem can be solved numerically using a conventional gradient-based optimization technique such as SQP. There are several efficient implementations of SQP available (see the subroutines NLPQL and NLPQLP written by Schittkowski (1985, 2004), for example).

\section{$5 \quad$ Numerical Examples}

To illustrate the concepts discussed in the preceding sections, we consider two examples. Example 1 is a problem with variable characteristic times. In Example 2, the characteristic times are fixed.

\section{Example 1. (Optimal Observation Times)}

Consider a process that evolves over the time horizon $[0,10]$ according to the following dynamical system:

$$
\begin{aligned}
\dot{x}_{1}(t) & =x_{2}(t), \\
x_{1}(0) & =0
\end{aligned}
$$

and

$$
\begin{aligned}
& \dot{x}_{2}(t)=-\zeta x_{2}(t)+u(t)-x_{1}(t) \\
& x_{2}(0)=0.2
\end{aligned}
$$

Our aim is to choose a control function $u(t)$ and system parameter $\zeta$ so that the system state $x_{1}$ is steered to- 
wards the target trajectory

$$
w(t)=4+\sin (2 t)+\exp (t / 5)
$$

Three observation time points are to be chosen when the distance between the system state and the target trajectory is minimal. In addition, control effort is to be conserved and the system state is subject to the following terminal constraint:

$$
0 \leq x_{1}(10) \leq 2
$$

To reflect these contrasting objectives, we consider a cost functional of the form

$$
\sum_{i=1}^{3}\left(w\left(\tau_{i}\right)-x_{1}\left(\tau_{i}\right)\right)^{2}+0.2 \int_{0}^{10} u^{2}(t) d t
$$

where $\tau_{1}, \tau_{2}$ and $\tau_{3}$ are the observation times. Our multiple characteristic time optimal control problem is to choose a control function $u(t)$, a system parameter $\zeta \in$ $[-1,1]$, and observation times $\tau_{1} \in[1,3], \tau_{2} \in[4,7]$ and $\tau_{3} \in[8,9]$ so that $(28)$ is minimized subject to the dynamics (23)-(26), the terminal state constraint (27), and $u(t) \in[-2,2]$ for all $t \in[0,10]$.

In applying the parametrization technique of Section 3, we allowed the control to switch once between each set of characteristic times and at the characteristic times themselves. The resulting optimization problem was solved using the subroutine NLPQLP with the function values and gradients constructed according to Algorithm 1. The relevant differential equations were solved using the LSODA solver (see Hindmarsh (1982)). It is important to note that, in accordance with Algorithm 1 , the auxiliary system and state system were solved together as one expanded system. Such an approach is not possible using the costate-based methods developed in Martin (1992) and Teo et al. (2002). Using these methods, the state system would need to be solved first, and then the state would need to be interpolated as the costate system is solved.

The optimal objective function value was found to be 17.3946, with $\zeta^{*}=-0.296$ and optimal observation times $\tau_{1}^{*}=2.7609, \tau_{2}^{*}=4.0000$ and $\tau_{3}^{*}=8.7511$. The optimal system state and optimal control structure are shown in Figures 1 and 2, respectively.

\section{Example 2. (Optimal Drug Administration)}

We consider the optimal control model of cancer chemotherapy formulated by Martin (1992). There are two state variables in the model: $v(t)$, the concentration of anti-cancer drug at the cancer site, and $N(t)$, the number of tumour cells. If we let $u(t)$ be the delivery rate of the anti-cancer drug and $y(t)=\ln (\theta / N(t))$, then

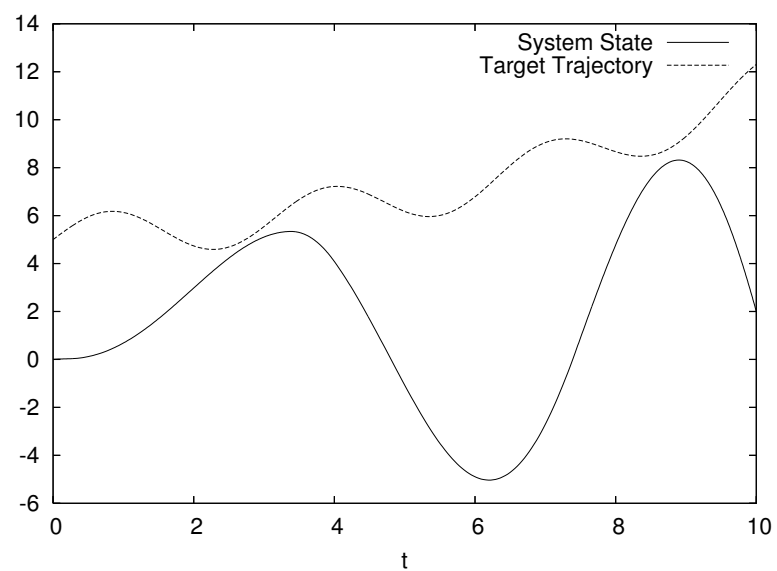

Fig. 1. The optimal system state for Example 1.

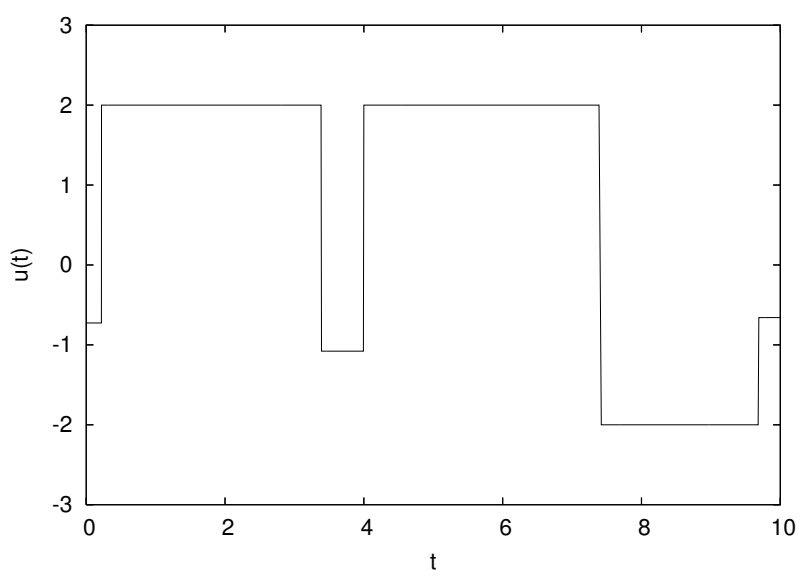

Fig. 2. The optimal control for Example 1.

the model dynamics are

$\begin{aligned} \dot{y}(t) & =-\lambda y(t)+k\left(v(t)-v_{\mathrm{th}}\right) H\left(v(t)-v_{\mathrm{th}}\right) \\ y(0) & =\ln \left(\theta / N_{0}\right)\end{aligned}$

and

$\dot{v}(t)=u(t)-\gamma v(t)$,

$v(0)=0$,

where $H(\cdot)$ denotes the Heaviside step function. The treatment starts at $t=0$ and ends at $t=T$. The constants $T, k, \lambda, \theta, N_{0}, \gamma$ and $v_{\text {th }}$ are model parameters.

Since anti-cancer drugs are highly toxic, restrictions are placed on the amount of drug that can be administered. These restrictions are expressed mathematically by the following two constraints on the drug concentration:

$$
0 \leq v(t) \leq v_{\max }, \quad \text { for all } t \in[0, T],
$$

and

$$
\int_{0}^{T} v(s) d s \leq v_{\mathrm{cum}}
$$


where $v_{\max }$ and $v_{\text {cum }}$ are constants. In addition, $M+1$ characteristic time points $0=\tau_{0}<\tau_{1}<\cdots<\tau_{M}<T$ are selected. The tumour size is required to decrease sufficiently between these characteristic times. This leads to the following set of multiple characteristic time constraints:

$$
y\left(\tau_{i}\right)-y\left(\tau_{i-1}\right)+\ln (\varepsilon) \geq 0, \quad i=1, \ldots, M,
$$

where $0<\varepsilon<1$. For full explanation of the model, see Martin (1992). We choose $n+1$ fixed time points $\left\{t_{i}\right\}_{i=0}^{n}$, where $t_{0}=0$ and $t_{n}=T$, and approximate the drug delivery rate by a constant $\sigma_{i}$ on the interval $\left[t_{i-1}, t_{i}\right)$, $i=1, \ldots, n$. Then, it is shown in Martin (1992) that the continuous time constraints (33) may be replaced by

$$
v\left(t_{i}\right) \leq v_{\max }, \quad i=1, \ldots, n
$$

We want to choose the drug delivery rate to minimize final tumour size. Thus, our multiple characteristic time optimal parameter selection problem is to choose $\boldsymbol{\sigma}=$ $\left[\sigma_{1}, \ldots, \sigma_{n}\right]^{T}$ to minimize $-y(T)$, subject to the dynamics (29)-(32), the constraints (34) and (36), and the multiple characteristic time constraints (35). The problem was solved numerically for different values of $n$ using the following parameter values: $T=84.0, \lambda=9.9 \times 10^{-4}$, $\gamma=0.27, k=8.4 \times 10^{-3}, v_{\mathrm{th}}=10.0, v_{\max }=50.0$, $v_{\text {cum }}=1100.0, \varepsilon=0.5, \theta=10^{12}, N_{0}=10^{10}, t_{i}=i T / n$, $i=0, \ldots, n$, and $\tau_{i}=i T / 4, i=0, \ldots, 3$. The subroutine NLPQLP was used for this purpose. Again, the required function values and gradients were constructed according to Algorithm 1, with LSODA used to solve the relevant differential equations. Initially, the number of control parameters was taken as $n=16$. This was subsequently doubled at each step until $n=128$. The tumour cell population after twelve weeks of treatment for this optimal scheme was $N(T)=3.2492 \times 10^{7}$. The treatment protocol and drug concentration for the optimal regime are shown in Figures 3 and 4, respectively.

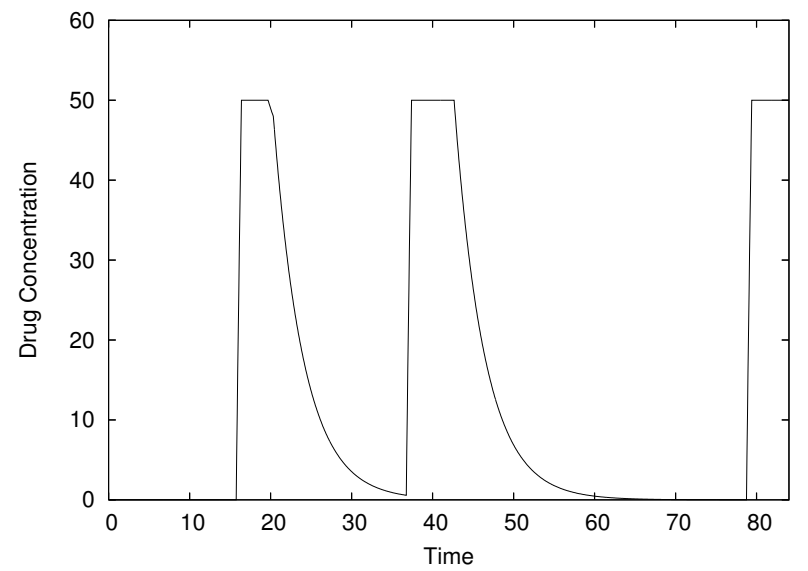

Fig. 3. The drug concentration for the optimal treatment protocol in Example 2.

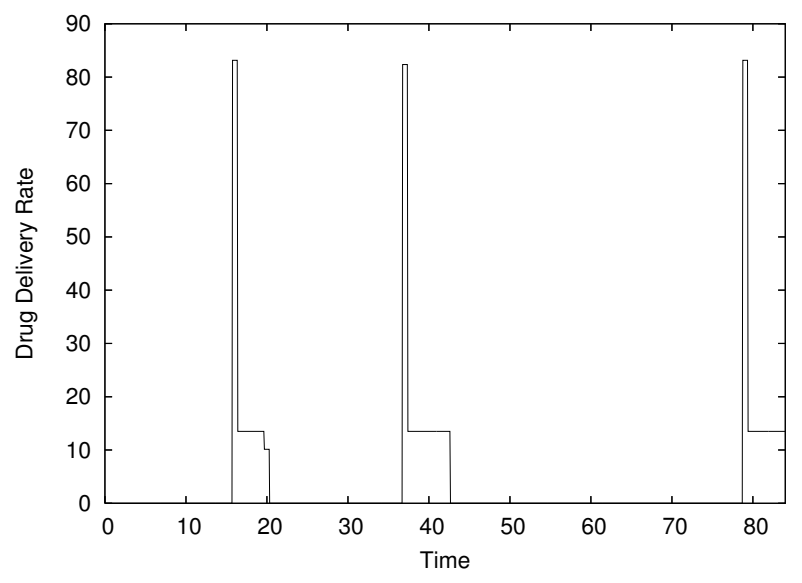

Fig. 4. The optimal treatment protocol in Example 2.

\section{Conclusion}

In this paper we have presented a computational procedure for solving optimal control problems with objective and constraint functionals depending on two or more characteristic times. Control parametrization and a time scaling transformation were applied to approximate this type of optimal control problem by a sequence of optimal parameter selection problems. Each of these optimal parameter selection problems can be viewed as a non-linear optimization problem and a new scheme for calculating the cost and constraint gradients was proposed. Using this scheme, standard gradient-based optimization techniques can be used to solve each approximate problem.

Interestingly and in contrast to the existing methods developed in Martin (1992), Martin \& Teo (1994) and Teo et al. (2003), the auxiliary system of differential equations used here does not contain any jump conditions. Moreover, as we discussed in the examples, this auxiliary system can be solved simultaneously with the state system. Thus, no interpolation of the state is necessary if a variable step size integration method is used to solve this expanded system. Efficiency, accuracy and simplicity are therefore enhanced. It is also worth recalling that the method proposed here is more efficient than than the costate-based method for problems with constraints outnumbering the optimization parameters. The cancer chemotherapy problem considered in Example 2 is of this type.

\section{References}

[1] Hindmarsh, A. (1982). Large ordinary differential equation systems and software. IEEE Control Systems Magazine, 2(4), 24-30.

[2] Kaya, C. Y. \& Noakes, J. L. (2003). Computational Method for Time-Optimal Switching Control. Journal of Optimization Theory and Applications, 117(1), 69-92.

[3] Lee, H. W. J., Teo, K. L., Rehbock, V., \& Jennings, L. S. (1997). Control parametrization enhancing technique 
for time optimal control problems. Dynamic Systems and Applications, 6, 243-262.

[4] Li, T. S., Livk, I. \& Ilievski, D. (2001). Influence of the Estimation Procedure on the Accuracy and Precision of Aluminium Trihydroxide Crystallization Kinetics from Dynamic Data. Industrial \& Engineering Chemistry Research, 40(22), 5005-5013.

[5] Livk, I., Pohar, C., \& Ilievski, D. (1999). Estimation of Batch Precipitation Kinetics by a Simplified Differential Method. AIChE Journal, 45(7), 1593-1596.

[6] Martin, R. B. (1992). Optimal Control Drug Scheduling of Cancer Chemotherapy. Automatica, 28(6), 1113-1123.

[7] Martin, R. B. \& Teo, K. L. (1994). Optimal control of drug administration in cancer chemotherapy. Singapore: World Scientific.

[8] Nocedal, J. \& Wright, S. J. (1999). Numerical Optimization. New York: Springer.

[9] Schittkowski, K. (1986). NLPQL: A fortran subroutine solving constrained nonlinear programming problems. Annals of Operations Research, 5, 485-500.

[10] Schittkowski, K. (2004). NLPQLP: A Fortran Implementation of a Sequential Quadratic Programming Algorithm with Distributed and Non-Monotone Line Search. User's Guide Version 2.0, University of Bayreuth.

[11] Teo, K. L., Goh, C. J., \& Wong, K. H. (1991). A unified computational approach to optimal control problems. Essex: Longman Scientific and Technical.

[12] Teo, K. L., Lee, W. R., Jennings, L. S., Wang, S. \& Liu, Y. (2002). Numerical solution of an optimal control problem with variable time points in the objective function. ANZIAM Journal, 43, 463-478.

[13] Vincent, T. L. \& Grantham, W. J. (1981). Optimality in parametric systems. New York: John Wiley. 